\title{
Relationship between Flow-mediated Endothelial Vasodilation and the Pulse Wave Velocity, and Cervical Carotid Artery Stenosis
}

\author{
Kazutaka SHIROKANE, , ${ }^{1,2}$ Tomonori TAMAKI, ${ }^{1}$ Kyongsong KIM, ${ }^{2}$ \\ Masato TSUCHIYA, ${ }^{3}$ Michio YAMAZAKI, ${ }^{1}$ and Akio MORITA ${ }^{4}$ \\ ${ }^{1}$ Department of Neurological Surgery, Tama Nagayama Hospital, Nippon Medical School, \\ Tama, Tokyo, Japan; \\ ${ }^{2}$ Department of Neurological Surgery, Chiba Hokusoh Hospital, Nippon Medical School, \\ Inzai, Chiba, Japan; \\ ${ }^{3}$ Mejiro-second Hospital, Fussa, Tokyo, Japan; \\ ${ }^{4}$ Nippon Medical School, Tokyo, Japan
}

\begin{abstract}
Carotid artery stenosis is elicited by atherosclerosis and is the main cause of cerebral thrombosis. Flowmediated endothelial vasodilation (FMD) can be measured noninvasively to assess vascular endothelial function related to atherosclerosis. The pulse wave velocity (PWV) is used to evaluate the vascular media involved in atherosclerosis. We investigated the relationship between these measurements in 75 consecutive patients with atherosclerotic cerebral thrombosis. They were assigned to three equal groups based on the severity of carotid artery stenosis on ultrasonograms. Group 1 had no stenosis, group 2 manifested moderate stenosis $(<60 \%)$, and group 3 presented with severe stenosis $(\geq 60 \%)$. We compared the FMD and PWV among the three groups. The PWV was significantly lower in group 1 than the other two groups. The FMD was significantly lower in group 3; it was significantly lower in group 2 than group 1 . There was an inverse correlation between the FMD and the severity of carotid artery stenosis. Our findings show that for assessing the severity of carotid artery stenosis, the FMD is more useful than the PWV.
\end{abstract}

Key words: carotid artery stenosis, endothelial dysfunction, ultrasonography, flow mediated dilation, pulse wave velocity

\section{Introduction}

Atherosclerosis of the cervical carotid artery plays an important role in ischemic cerebrovascular disease; it is also a reliable marker of systemic atherosclerosis. ${ }^{1-3)}$ The pulse wave velocity (PWV) is associated with major cardiovascular risk factors; it is used to assess the function of the medial membrane. Although evaluation of the PWV is a noninvasive method to diagnose systemic atherosclerotic changes, it does not yield information on the severity and progression of cervical carotid artery stenosis. ${ }^{4)}$

Endothelial cells play an important role in the protection from atherosclerotic progression. Impairment of endothelial function may result in the promotion

Received September 12, 2019; Accepted March 13, 2020

Copyright $\odot 2020$ by The Japan Neurosurgical Society This work is licensed under a Creative Commons AttributionNonCommercial-NoDerivatives International License. of atherosclerotic changes and in plaque formation. ${ }^{5)}$ Measurement of flow-mediated endothelial vasodilation (FMD) in the brachial artery is a commonly used, noninvasive method to assess endothelial cell function (Fig. 1) ${ }^{6-11)}$ related to atherosclerotic changes such as carotid elongation, ${ }^{12)}$ cardiovascular risk, ${ }^{5-9)}$ and cortical cerebral infarction. ${ }^{11)}$ A meta-analysis performed by Inaba et al. ${ }^{5)}$ showed that impairment of the brachial FMD was not only associated with known cardiovascular risk factors but also significantly associated with future cardiovascular events.

We investigated the relationship between PWV and FMD measurements and the degree of cervical carotid artery stenosis ranging from mild to severe.

\section{Materials and Methods}

From among 186 consecutive patients with cerebral thrombotic infarction who were at least 60 years old at the time of hospitalization in Tama Nagayama Hospital 
Measurement of flow-mediated endothelial vasodilation in the brachial artery

(1) Measurement of the brachial artery diameter at rest

(2) Blood-flow blockage with a pneumatic cuff inflated for $5 \mathrm{~min}$ to $50 \mathrm{mmHg}$ above the systolic pressure.

(3) Releasing the cuff to elicit reactive hyperemia.
a Immediate increase in the blood flow
b Increase in the vessel wall shear stress on endothelial cells
c Vasodilator substances released from endothelial cells
d Vasodilator substances act on vascular smooth cells
e Dilation of the brachial artery

(4) Measurement of the maximum diameter after blood-flow blockage

$\mathrm{FMD} \%=[($ maximum diameter after blood-flow blockage - artery diameter at rest $) /$ artery diameter at rest] $\mathrm{x} 100$

Fig. 1 Measurement of flow-mediated endothelial vasodilation in the brachial artery.

between April 2009 and March 2012, we selected 75 for inclusion in this study. They were 52 males and 23 females; their mean age was 65 years (range 60-79 years) (Table 1). Patients unable to cooperate, patients with cerebral hemorrhage, transient ischemic attacks, subarachnoid hemorrhage, embolic stroke secondary to heart disease, brain tumors, dementia, occlusion of the cervical artery, and in postoperative status were excluded from our study.

Cerebral thrombosis was diagnosed in patients with rapid-onset brain infarction whose symptoms were present for more than $24 \mathrm{~h}$ and who manifested no other vascular occlusion. ${ }^{4)}$ Based on their clinical characteristics and brain computed tomography (CT)- and/ or findings, the infarctions were classified as cortical or subcortical. ${ }^{4)}$ Cortical infarction was recorded when there was clinical evidence of cortical deficits and when CT- and/or magnetic resonance imaging (MRI) scans showed an infarct involving the cerebral cortex in the carotid artery territory without evidence of cardiogenic embolism. Subcortical infarction was recorded when CT- and/or MRI studies showed that a small infarct in the subcortical carotid artery territory was responsible for the symptoms. Patients with combined cortical and subcortical involvement on imaging studies were assigned to the cortical infarction group.
The 75 included patients were assigned to three equal groups. Group 1 manifested no-, group 2 moderate $(<60 \%)$-, and group 3 severe $(\geq 60 \%)$ carotid artery stenosis. We compared the FMD and PWV among the three groups.

To evaluate maximum cervical carotid artery stenosis, we applied the area method using cervical echo findings. ${ }^{1)}$ We performed high-resolution B-mode ultrasonography on a 7.5-MHz duplex scanner (Hitachi EUB-555, Hitachi, Japan). We defined carotid atheromatous plaques as lesions with a carotid [intima media thickness (IMT) $>1.1 \mathrm{~mm}$ ]. To determine the degree of right- and left-sided cervical carotid artery stenosis we recorded the area involved by stenosis.

To assess endothelial function we determined the FMD on a high-resolution ultrasound apparatus featuring a $7.5-\mathrm{MHz}$ linear array transducer and on-line computer-assisted semi-automatic analysis software (EF, Unex Co. Ltd, Nagoya, Japan). We applied the guidelines of the International Brachial Artery Reactivity Task Force. ${ }^{13)}$ Since the FMD is affected by factors such the body temperature, food, drugs, and sympathetic stimuli, we measured it in the early morning, before a meal, and after a 10-min rest when the systolic blood pressure was $160 \mathrm{mmHg}$ and the pulse 100 beats per min or less. The subjects 
Table 1 Patient characteristics

\begin{tabular}{|c|c|c|c|c|}
\hline \multirow{2}{*}{$\begin{array}{l}\text { Degree of cervical } \\
\text { carotid artery } \\
\text { stenosis }\end{array}$} & \multicolumn{3}{|c|}{ Carotid artery stenosis } & \multirow[b]{2}{*}{ Difference } \\
\hline & $\begin{array}{c}\text { None } \\
\text { (group 1) }\end{array}$ & $\begin{array}{c}<60 \% \\
\text { (group 2) }\end{array}$ & $\begin{array}{c}\geq 60 \% \\
\text { (group 3) }\end{array}$ & \\
\hline Patients & 25 & 25 & 25 & NS \\
\hline Age (years) & $67 \pm 7$ & $69 \pm 6$ & $72 \pm 6$ & NS \\
\hline $\begin{array}{l}\text { Gender } \\
\text { (male/female) }\end{array}$ & $16 / 9$ & $17 / 8$ & $19 / 6$ & NS \\
\hline \multicolumn{5}{|l|}{ Medication } \\
\hline HT & 21 & 23 & 24 & NS \\
\hline HL & 17 & 15 & 19 & NS \\
\hline $\mathrm{DM}$ & 8 & 16 & 17 & $P<0.05$ \\
\hline Smoking & 19 & 22 & 24 & NS \\
\hline \multicolumn{5}{|l|}{ Stroke types } \\
\hline $\begin{array}{l}\text { Cortex CI/ } \\
\text { subcortex CI }\end{array}$ & $2 / 23$ & $10 / 15$ & $25 / 0$ & $P<0.05$ \\
\hline $\begin{array}{l}\text { Cervical carotid } \\
\text { artery stenosis (\%) }\end{array}$ & & $36 \pm 27$ & $85 \pm 9$ & \\
\hline
\end{tabular}

were instructed to fast for at least $4 \mathrm{~h}$ and to abstain from smoking and from ingesting alcohol, caffeine, and antioxidant vitamins for at least $12 \mathrm{~h}$. They sat in a quiet, dark, air-conditioned room $\left(22-25^{\circ} \mathrm{C}\right)$ for 15 min before FMD measurements were performed by a single examiner. The two echo probes were placed in the brachial artery and the vessel diameter between the two probes was recorded. First, the brachial artery diameter $5-10 \mathrm{~cm}$ above the elbow at rest was measured (Fig. 1). For blood-flow blockage we placed a pneumatic cuff and inflated it for $5 \mathrm{~min}$ to $50 \mathrm{mmHg}$ above the systolic pressure. After deflating the cuff to elicit reactive hyperemia, the brachial artery diameter was recorded continuously for $2 \mathrm{~min}$. We also measured the maximum diameter of the radial artery after blood-flow blockage. The FMD was calculated with the formula

$\mathrm{FMD} \%=\left[\begin{array}{l}\text { Maximum diameter after blood- } \\ \text { flow blockage }- \\ \frac{\text { Artery diameter at rest }}{\text { Artery diameter at rest }}\end{array}\right] \times 100$.

We evaluated the PWV/ankle brachial pressure index (ABI) using the AT-form PWV/ABI (Nippon Colin Co., Ltd., Komaki, Japan ${ }^{4)}$ and simultaneously measured the bilateral brachial and tibial artery pressure waveforms using a volume-plethysmographic apparatus. The brachial-ankle PWV was calculated based on the time required for the waveform to travel between the right arm and both ankles. The distance between the right arm and the ankle was estimated based on the patient's height. We used the mean value of our bilateral measurement data. None of our 75 patients had an ABI below 0.8.

Our findings are expressed as the mean \pm SD. All statistical analyses were performed using StatView version 4.0 software (Abacus Concepts Inc., Berkeley, CA, USA). For parametric data analysis we used the unpaired $t$-test for 2-group comparisons. For 3-group comparisons we applied one-way analysis of variance with Fisher's least-significant difference post-hoc test. For non-parametric data analysis, we performed the Mann-Whitney $U$-test for comparisons. Differences of $P<0.05$ were considered statistically significant.

\section{Results}

There was no statistically significant difference in our patients' age, gender, and smoking habit. The number of patients with diabetes mellitus (DM) was significant lower in group 1 than the other groups. Of the 75 patients $37(49.3 \%)$ presented with cortical- and 38 (50.7\%) with subcortical infarction. The number of patients with cortical infarction was significantly higher in group 3; subcortical infarction was significantly lower in group 1 .

As shown in Fig. 2, the PWV was significantly lower in group $1(1702 \pm 349 \mathrm{~cm} / \mathrm{s})$ than group 2 $(2103 \pm 427 \mathrm{~cm} / \mathrm{s})$ and group $3(2225 \pm 384 \mathrm{~cm} / \mathrm{s})$ 


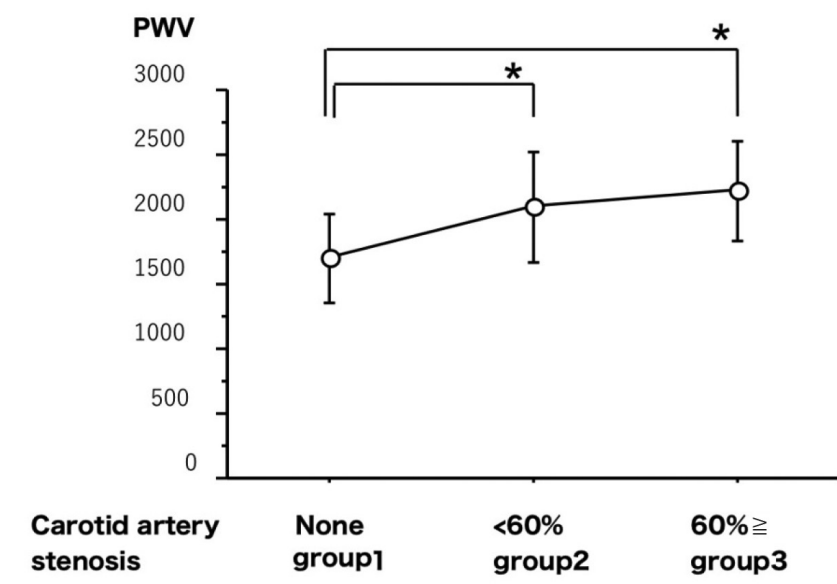

Fig. 2 Pulse wave velocity (PWV) recorded in patients with cervical carotid artery stenosis. The PWV in patients without- was significantly lower than in patients with stenosis. ${ }^{*} P<0.05$.

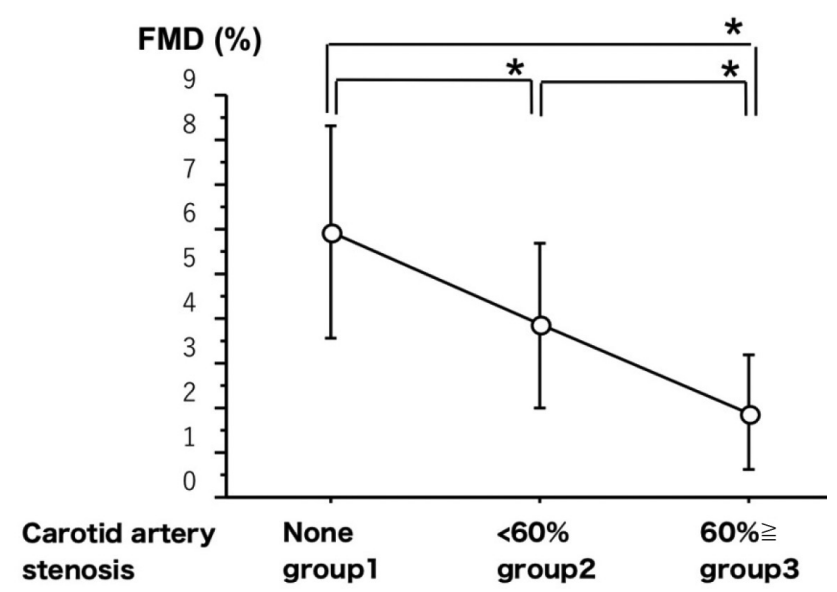

Fig. 3 Flow-mediated endothelial vasodilatation (FMD) recorded in patients with cervical carotid artery stenosis. The FMD was significantly larger in patients without- than with moderate to severe stenosis (group 1 vs groups 2 and 3 ).

$(P<0.05)$. The FMD was significantly greater in group $1(5.9 \pm 2.3 \%)$ than group $2(3.8 \pm 1.8 \%)$ and group $3(1.9 \pm 1.3 \%)$ (all, $P<0.05)$; it was significantly greater in group 2 than group 3 (Fig. 3 ).

Of the 25 group 3 patients, 24 subsequently underwent carotid endarterectomy (CEA). There were no complications related to systemic arteriosclerosis such as ischemic heart disease during the perioperative period.

\section{Discussion}

The PWV is associated with major cardiovascular risk factors and a predictor of the prognosis of patients with end-stage renal failure and hypertension. ${ }^{7)}$
According to Tamaki et al., ${ }^{4)}$ it is associated with carotid atherosclerosis but not with the severity of cervical carotid artery stenosis. However, it is correlated with the carotid IMT in patients with type 2 DM. ${ }^{14)}$ We found a correlation between the PWV and carotid atherosclerosis but not with the severity of cervical carotid artery stenosis, although 24 of our group 3 patients manifested severe cervical carotid artery stenosis requiring CEA. Assessment of the PWV is useful for the noninvasive evaluation of systemic atherosclerotic changes. However, it does not shed light on the severity and progression of cervical carotid artery stenosis, factors needed to predict the risk for a cerebral stroke. ${ }^{1,2)}$

Whether the FMD of the cervical carotid artery is a predictor of IMT worsening remains controversial. ${ }^{11-13)} \mathrm{A}$ meta-analysis reported by Lorenz et al. ${ }^{15)}$ failed to prove the association between IMT progression and cardiovascular risk in the general population. Juonala et al. ${ }^{16)}$ reported that in young healthy adults, the FMD was inversely associated with the cervical carotid IMT; the number of risk factors was correlated with increased IMT in subjects with an anomalous FMD. These studies suggest that systemic endothelial function may affect the association between risk factors and atherosclerosis. The FMD also predicted changes in the number of cervical carotid artery plaques. ${ }^{17)}$ Such results suggest that cervical carotid artery stenosis, indicative of an advanced stage of epithelial dysfunction, may be related with the FMD and that the FMD may alert to the progression of cervical carotid artery stenosis.

We enrolled patients with atherosclerotic cerebral thrombosis whose atherosclerosis seemed to be advanced; patients with severe carotid artery stenosis were also included. We assessed the relationship between the FMD and the degree of cervical carotid artery stenosis requiring CEA rather than the carotid IMT. We found that the FMD was significantly smaller in patients with severe cervical carotid artery stenosis (group 3) than in the other groups. Therefore, we think that FMD assessment is informative because it yields indirect qualitative information on not only the coronary- but also the cervical carotid artery.

The degree of stenosis and a cerebral infarct due to cervical carotid artery stenosis rendered some of our patients eligible for surgery. If the FMD can predict cerebral infarction related cervical carotid artery stenosis and alert to its progression, it can be used to identify patients needing CEA for the prevention of later cerebral infarction. Our crosssectional study revealed that the severity of cervical carotid artery stenosis was correlated with the FMD. However, longitudinal prospective studies are needed 
to confirm that the FMD reflects the progression of cervical carotid artery stenosis and that it alerts to the danger of cerebral infarction.

We document that there is a stronger relationship between cervical carotid artery stenosis and the FMD than the PWV. While the PWV is useful for differentiating between patients with- and without stenosis, it is not a suitable parameter for the quantitative evaluation of stenosis. Because the PWV reflects the compliance of the blood vessel wall, it can be used to detect deterioration in the function of the media. The FMD, on the other hand, reflects the release of nitric oxide in the vascular endothelium; it can be used to check for deterioration of the inner membrane. As cervical carotid artery stenosis results in thickening of the intima, it makes sense to suggest that the FMD is a better parameter than the PWV for the evaluation of cervical carotid artery stenosis.

Our study has some limitations. The number of patients was relatively small. We encountered no perioperative complications related to systemic arteriosclerosis although 24 of our group 3 patients required CEA. While the relationship between the FMD, an index of arteriosclerosis, and systemic arteriosclerotic complications during the perioperative period is very interesting, it was not examined in this study. Although measurement of the FMD is noninvasive, highly versatile, and useful for managing lifestylerelated diseases, it requires pre-procedural fasting and patient medications must be taken into account. Environmental factors also affect FMD measurements and patient cooperation is required. Cervical carotid artery plaques may harbor unstable components such as a lipid core and intraplaque hemorrhage ${ }^{18-20)}$ that may increase the risk for cerebral infarction. We did not evaluate the relationship between the plaque makeup and the FMD. Others ${ }^{6,8,21)}$ reported that in patients whose FMD measurements indicated late, albeit maximal dilation, the cardiovascular risk profile was worse and the incidence of carotid atherosclerosis was higher than in patients with early dilation. Therefore, not only the magnitude of dilation but also its timing is predictive of cardiovascular risk. Studies are underway to address these issues. Our cross-sectional study was performed to investigate the relationship between the FMD and the severity of cervical carotid stenosis. Longitudinal studies are needed to determine whether stenotic progression can be predicted by the FMD.

\section{Conclusion}

The FMD is inversely correlated with the severity of carotid artery stenosis in patients with atherosclerotic cerebral thrombosis. The PWV, on the other hand, reflects only the absence/presence of carotid stenosis. We found that for assessing the severity of carotid stenosis, FMD- were superior to PWV measurements.

\section{Conflicts of Interest Disclosure}

None.

\section{References}

1) MRC European Carotid Surgery Trial: interim results for symptomatic patients with severe $(70-99 \%)$ or with mild (0-29\%) carotid stenosis. European Carotid Surgery Trialists' Collaborative Group. Lancet 337: 1235-1243, 1991

2) North American Symptomatic Carotid Endarterectomy Trial Collaborators, Barnett HJM, Taylor DW, et al.: Beneficial effect of carotid endarterectomy in symptomatic patients with high-grade carotid stenosis. $N$ Engl J Med 325: 445-453, 1991

3) Bruno RM, Bianchini E, Faita F, Taddei S, Ghiadoni L: Intima media thickness, pulse wave velocity, and flow mediated dilation. Cardiovasc Ultrasound 12: 34, 2014

4) Tamaki T, Keiko S, Shinkichi H, Yoji N, Akira T: Carotid atherosclerosis and arterial peripheral pulse wave velocity in cerebral thrombosis. J Clin Neurosci 13: 45-49, 2006

5) Inaba Y, Chen JA, Bergmann SR: Prediction of future cardiovascular outcomes by flow-mediated vasodilatation of brachial artery: a meta-analysis. Int J Cardiovasc Imaging 26: 631-640, 2010

6) Irace C, Tripolino C, Scavelli FB, Carallo C, Gnasso A: Brachial low-flow-mediated constriction is associated with delayed brachial flow-mediated dilation. J Atheroscler Thromb 23: 355-363, 2016

7) Irace C, De Rosa S, Tripolino C, et al.: Delayed flowmediated vasodilation and critical coronary stenosis. J Investig Med 66: 1-7, 2018

8) Irace C, Padilla J, Carallo C, Scavelli F, Gnasso A: Delayed vasodilation is associated with cardiovascular risk. Eur J Clin Invest 44: 549-556, 2014

9) Kuvin JT, Patel AR, Sliney KA, et al.: Peripheral vascular endothelial function testing as a noninvasive indicator of coronary artery disease. $J \mathrm{Am}$ Coll Cardiol 38: 1843-1849, 2001

10) Celermajer DS, Sorensen KE, Gooch VM, et al.: Non-invasive detection of endothelial dysfunction in children and adults at risk of atherosclerosis. Lancet 340: 1111-1115, 1992

11) Lind L, Nylander R, Johansson L, Kullberg J, Ahlstrom H, Larsson EM: Endothelium-dependent vasodilation is related to the occurrence of cortical brain infarcts at MR imaging: The Prospective Investigation of the Vasculature in Uppsala Seniors (PIVUS) study. Clin Physiol Funct Imaging 37: 194-197, 2017

12) Baracchini C, Farina F, Tonello S, et al.: Endothelial dysfunction in carotid elongation. J Neuroimaging 23: 18-20, 2013 
13) Corretti MC, Anderson TJ, Benjamin EJ, et al.: Guidelines for the ultrasound assessment of endothelialdependent flow-mediated vasodilation of the brachial artery: a report of the International Brachial Artery Reactivity Task Force. J Am Coll Cardiol 39: 257-265, 2002

14) Taniwaki H, Kawagishi T, Emoto M, et al.: Correlation between the intima-media thickness of the carotid artery and aortic pulse-wave velocity in patients with type 2 diabetes. Vessel wall properties in type 2 diabetes. Diabetes Care 22: 1851-1857, 1999

15) Lorenz MW, Polak JF, Kavousi M, et al.: Carotid intima-media thickness progression to predict cardiovascular events in the general population (the PROG-IMT collaborative project): a meta-analysis of individual participant data. Lancet 379: 2053-2062, 2012

16) Juonala M, Viikari JS, Laitinen $\mathrm{T}$, et al.: Interrelations between brachial endothelial function and carotid intima-media thickness in young adults: the cardiovascular risk in young Finns study. Circulation 110: 2918-2923, 2004

17) Lind L: Flow-mediated vasodilation was found to be an independent predictor of changes in the carotid plaque status during a 5-year follow-up. J Atheroscler Thromb 21: 161-168, 2014
18) Yamada K, Kawasaki M, Yoshimura S, et al.: Evaluation of symptomatic carotid plaques by tissue characterization using integrated backscatter ultrasound and magnetic resonance imaging. Cerebrovasc Dis 31: 305-312, 2011

19) Polak JF, Shemanski L, O'Leary DH, et al.: Hypoechoic plaque at US of the carotid artery: an independent risk factor for incident stroke in adults aged 65 years or older. Cardiovascular Health Study. Radiology 208: 649-654, 1998

20) Redgrave JN, Lovett JK, Gallagher PJ, Rothwell PM: Histological assessment of 526 symptomatic carotid plaques in relation to the nature and timing of ischemic symptoms: the Oxford plaque study. Circulation 113: 2320-2328, 2006

21) Tripolino C, Gnasso A, Carallo C, Scavelli FB, Irace C: Difference in carotid artery elasticity in subjects with different brachial artery kinetic of vasodilatation. J Hum Hypertens 30: 493-497, 2016

Address reprint requests to: Tomonori Tamaki, MD, PhD, Department of Neurological Surgery, Tama Nagayama Hospital, Nippon Medical School, 1-7-1 Nagayama, Tama, Tokyo, Japan.

e-mail: tamakito@nms.ac.jp 